\title{
Author Correction: Foldamers wave to the ribosome
}

\author{
Alanna Schepartz
}

Correction to: Nature Chemistry https://doi.org/10.1038/s41557-018-0036-5, published online 22 March 2018.

In the News \& Views 'Foldamers wave to the ribosome', multiple changes to the main text were required. In paragraph 2 "whose monomer sequence is truly defined" should have read "whose monomer sequence is both diverse and truly defined" and "Truly sequencedefined..." should have read "Diverse and truly sequence defined...”. In the penultimate paragraph, 'appendages 5-9' should have been "appendages 5 and 7-9"; in the sentence starting "Based on these criteria..., QPQ should have been QQQ and vice versa; and the whole sentence starting "What is interesting..." needed revising to "What is interesting is that an alternating sequence with a terminal $P$ monomer appears essential: PQPQ is unfolded and an initiator tRNA carrying PQPQ-Gly-Phe initiates translation, whereas QPQPQ is folded and does not." Furthermore, the structures in Fig. 1a have been replaced to correct several errors in the chemical structures arising from missing $-\mathrm{CH}_{2}-$ groups. The table in Fig. $1 \mathrm{~b}$ also required changes: in the last two columns of row two, 'Fast' and 'No' should have read 'Slow and 'Yes', respectively; in the first column of row three, 'ND' should have been a tick; and in the last two columns of row four, 'Slow' and 'Yes' should have read 'Fast' and 'No', respectively. Finally, a new reference 'Milon, P. et al. EMBO Rep. 11, 312-316 (2010).' has been added to the reference list at number 13 and cited in the fifth paragraph, the references list has been renumbered accordingly.

All of these changes have been made to the online versions of this News \& Views.

a

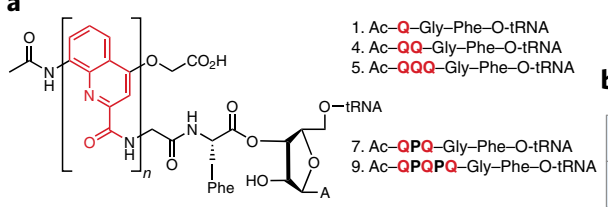

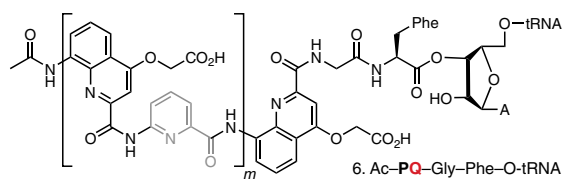

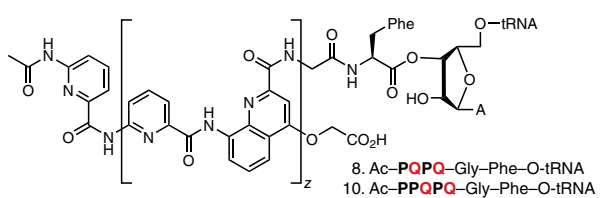

a

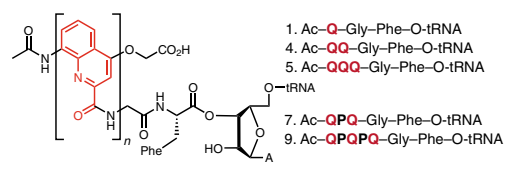

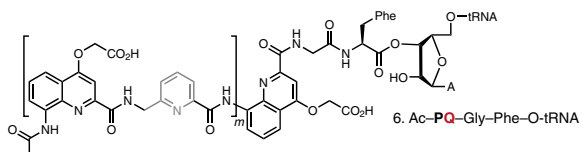

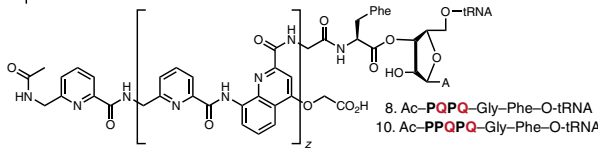

Original

b
\begin{tabular}{|c|c|c|c|c|}
\hline $\begin{array}{c}\text { Foldamer } \\
\text { appendage }\end{array}$ & $\begin{array}{c}\text { Initiation } \\
\text { (foldamer-peptide) }\end{array}$ & $\begin{array}{c}\text { Initiation } \\
\text { (foldamer-protein) }\end{array}$ & $\begin{array}{c}\text { Amide NH } \\
\text { exchange }\end{array}$ & $\begin{array}{c}\text { Diastereotopic } \\
\text { 'H NMR }\end{array}$ \\
\hline Ac-QQ-Gly-Phe (4) & $\checkmark$ & ND & - & - \\
\cline { 2 - 4 } Ac-QQQ-Gly-Phe (5) & $\times$ & ND & Fast & \multirow{2}{*}{ No } \\
\hline Ac-PQ-Gly-Phe (6) & ND & $\checkmark$ & - & - \\
\hline Ac-QPQ-Gly-Phe (7) & $\checkmark$ & ND & Slow & Yes \\
\hline Ac-PQPQ-Gly-Phe (8) & $\checkmark$ & $\checkmark$ & Fast & Partial \\
\hline Ac-QPQPQ-Gly-Phe (9) & $\times$ & ND & Slow & Yes \\
\hline Ac-PPQPQ-Gly-Phe (10) & $\checkmark$ & $\checkmark$ & - & - \\
\hline
\end{tabular}

\section{Corrected}

\begin{tabular}{|c|c|c|c|c|}
\hline $\begin{array}{c}\text { Foldamer } \\
\text { appendage }\end{array}$ & $\begin{array}{c}\text { Initiation } \\
\text { (foldamer-peptide) }\end{array}$ & $\begin{array}{c}\text { Initiation } \\
\text { (foldamer-protein) }\end{array}$ & $\begin{array}{l}\text { Amide NH } \\
\text { exchange }\end{array}$ & $\begin{array}{c}\text { Diastereotopic } \\
{ }^{1} \mathrm{H} \text { NMR }\end{array}$ \\
\hline Ac-QQ-Gly-Phe (4) & $\checkmark$ & ND & - & - \\
\hline Ac-QQQ-Gly-Phe (5) & $x$ & ND & Slow & Yes \\
\hline Ac-PQ-Gly-Phe (6) & $\checkmark$ & $\checkmark$ & - & - \\
\hline Ac-QPQ-Gly-Phe (7) & $\checkmark$ & ND & Fast & No \\
\hline Ac-PQPQ-Gly-Phe (8) & $\checkmark$ & $\checkmark$ & Fast & Partial \\
\hline Ac-QPQPQ-Gly-Phe (9) & $x$ & ND & Slow & Yes \\
\hline Ac-PPQPQ-Gly-Phe (10) & $\checkmark$ & $\checkmark$ & - & - \\
\hline
\end{tabular}

Fig. 1| Original and corrected.

Published online: 10 July 2018

https://doi.org/10.1038/s41557-018-0101-0 